\title{
Blow up results for a viscoelastic Kirchhoff-type equation with logarithmic nonlinearity and strong damping
}

\author{
Jorge Ferreira, Erhan Pişkin, Nazli Irkil, Carlos Raposo
}

\begin{abstract}
A Kirchhoff equation type with memory term competing with a logarithmic source is considered. By using potential well theory, we obtained the global existence of solution for the initial data in a stability set created from Nehari Manifold and prove blow up results for initial data in the instability set.
\end{abstract}

\section{INTRODUCTION}

We are investigating the following viscolelastic Kirchhoff type problem with logarithmic nonlinearity, for $(x, t) \in \Omega \times R^{+}$,

$$
\begin{array}{r}
u_{t t}-M\left(\|\nabla u\|^{2}\right) \Delta u+\int_{0}^{t} g(t-s) \Delta u(s) \mathrm{d} s-\Delta u_{t}=|u|^{p-2} u \ln |u|, \\
u(x, 0)=u_{0}(x), u_{t}(x, 0)=u_{1}(x), x \in \Omega, \\
u(x, t)=0, x \in \partial \Omega \times R^{+},
\end{array}
$$

where $\Omega$ is a bounded domain in $R^{n}, n \geq 1$ with smooth boundary $\partial \Omega$. $M(s)=\alpha+\beta s^{\gamma}(\gamma, s \geq 0)$, specially, we take $a=\beta=1$. We impose some conditions to be specified on the kernel function $g(t)$.

This kind of wave equation (1) is called of Kirchhoff type in reason of the one-dimensional nonlinear equation (4) proposed by Kirchhoff [10] (1883),

$$
\frac{\partial^{2} u}{\partial t^{2}}-\left(\frac{\tau_{0}}{m}+\frac{k}{2 m L} \int_{0}^{L}\left(\frac{\partial u}{\partial x}\right)^{2} \mathrm{~d} x\right) \frac{\partial^{2} u}{\partial x^{2}}=0,
$$

where $\tau_{0}$ is the initial tension, $m$ the mass of the string and $k$ the Young's modulus of the material of the string. This model, in connection with some problems in nonlinear elasticity, describes small vibrations of a stretched

2020 Mathematics Subject Classification. Primary: 35A01; Secondary: 35B44.

Key words and phrases. Existence, blow up, Kirchhoff-type equation with memory, logarithmic nonlinearity.

Full paper. Received 13 April 2021, revised 28 July 2021, accepted 16 September 2021, available online 24 September 2021. 
string of the length $L$ when only the transverse component of the tension is considered.

In [18], was proved existence and the energy decay estimate of global solutions for a extensible beam equation of Kirchhoff type with internal damping $\left|u_{t}\right|^{p-1} u_{t}$ and source term $|u|^{q-1} u$ given by

$$
\begin{array}{r}
u_{t t}+\Delta^{2} u+M\left(\left.|| \nabla u\right|^{2}\right)(-\Delta u)+\left|u_{t}\right|^{p-1} u_{t}=|u|^{q-1} u \quad \text { in } \Omega \times(0, T), \\
u(x, 0)=u_{0}(x), \quad u_{t}(x, 0)=u_{1}(x), \quad x \in \Omega, \\
u(x, t)=\frac{\partial u}{\partial \eta}(x, t)=0, \quad x \in \partial \Omega, t \geq 0,
\end{array}
$$

where $p \geq 1, q>1$ are real numbers, $\Omega$ be a bounded domain in $\mathbb{R}^{n}$ with smooth boundary $\partial \Omega$ and $M(s)$ is a continuous function on $[0,+\infty)$.

The following equation

$$
\left|u_{t}\right|^{\rho} u_{t t}-M\left(\|\Delta u\|^{2}\right) \Delta^{2} u+\Delta^{2} u_{t t}-\int_{0}^{t} h(t-s) \triangle^{2} u(s) \mathrm{d} s=|u|^{\gamma-1} u \ln |u|^{k}
$$

has been considered by Boulaaras et al. in [4]. They established polynomial decay rate estimates results of solutions. In presence of delay term with $\gamma=1$ the equation (8) has been studied by Mezouar et al. in [17]. They proved the global existence of weak solutions and the uniform decay of the energy is derived.

In the absence of kernel function (that is $g=0$ ) and by taking $M(s)=1$ and $p=2$, the equation (1) can be recorded in the form

$$
u_{t t}-\Delta u-\Delta u_{t}=u \ln |u| \text {. }
$$

Logarithmic nonlinearity term appears frequently in partial differential equations due to their wide application in physics and other applied sciences. Problems like equation (9) is encountered naturally in quantum mechanics, inflation cosmolog, supersymmetric field theories, and a lot of different areas of physics such as, optics, geophysics and nuclear physics $[5,6,11,13]$. With all those specific meaning in physics, the mathematical behaviour of solution to the problem of evolution equation with such logarithmic type nonlinearity takes lots of attention by many mathematicians. We also mention some related mathematical work involving the logarithmic term in the literature see, for example, $[7-9,12,15]$ and references therein.

When $M(s)=1, g \neq 0$, then these problems turn into viscoelastic wave equation with logarithmic source term. This type problems have been handled carefully by many authors in several works $[2,16,19,21,25]$.

In the present paper, we dedicate our study to problem (1)-(3). The structure of the work is as follows: To facilitate the description, firstly in section 2 we present the potential well. In Section 3 we present hypotheses and lemmas which will need throughout in our proof of existence of solution and 
blow up in finite time. In Section 4 and section 5, respectively, we establish the global existence and blow up results.

\section{Potential well}

As standard, $\|\cdot\|_{2}$ and $\|\cdot\|_{p}$ denotes the usual $L^{2}(\Omega)$ norm and $L^{p}(\Omega)$ norm, respectively. We denote by $C$ and $C_{i}(i=1,2, \ldots)$ various positive constants. We will use the Standart Lebesgue Space $L^{2}(\Omega)$ with the inner product and the norm. The inner product can take as

$$
\langle u, v\rangle=\int u(x) v(x) \mathrm{d} x
$$

and the norm is defined as

$$
\|u\|_{2}=\langle u, u\rangle^{\frac{1}{2}} .
$$

It is well known that the energy of a PDE system is, in some sense, split into kinetic and potential energy. Following the idea of Y. Ye [24] we are able to construct a set of stability as follows. We will prove that there is a valley or a "well" of depth $d$ created in the potential energy. If this height $d$ is strictly positive, we find that, for solutions with initial data in the "good part" of the well, the potential energy of the solution can never escape the well. In general, it is possible for the energy from the source term to cause the blow-up in finite time.

For $u \in H_{0}^{1}(\Omega)$ we define the functional

$$
\mathcal{J}(\lambda u)=\frac{\lambda^{2} \beta}{4(\gamma+1)}\|u\|^{2(\gamma+1)}-\frac{\lambda}{p} \int_{\Omega} u^{p} \ln |u| \mathrm{d} x, \quad 0<\lambda \leq 1 .
$$

Associated with the $\mathcal{J}$ we have the well known Nehari Manifold given by

$$
N \stackrel{\text { def }}{=}\left\{u \in H_{0}^{1}(\Omega) / 0:\left[\frac{d}{d \lambda} J(\lambda u)\right]_{\lambda=1}=0\right\} .
$$

From (10) we get

$$
\frac{\mathrm{d}}{\mathrm{d} \lambda} \mathcal{J}(\lambda u)=\frac{\lambda \beta}{2(\gamma+1)}\|u\|^{2(\gamma+1)}-\frac{1}{p} \int_{\Omega} u^{p} \ln |u| \mathrm{d} x,
$$

then

$$
\mathcal{N} \stackrel{\text { def }}{=}\left\{u \in H_{0}^{1}(\Omega) /\{0\}: \frac{\lambda \beta}{2(\gamma+1)}\|u\|^{2(\gamma+1)}=\frac{1}{p} \int_{\Omega} u^{p} \ln |u| \mathrm{d} x\right\} .
$$

We define as in the Mountain Pass theorem due to Ambrosetti and Rabinowitz [3],

$$
d=\inf _{\left\{u \in H_{0}^{1}(\Omega) / 0\right\}} \sup _{0 \leq \lambda} J(\lambda u)
$$


It is well-known that the depth of the well $d$ is a strictly positive constant, see [[22], Theorem 4.2], and

$$
d=\inf _{u \in \mathcal{N}} \mathcal{J}(u)
$$

In fact, in our problem, the solution of $\frac{\mathrm{d}}{\mathrm{d} \lambda} \mathcal{J}(\lambda u)=0$ is

$$
\lambda_{*}=\left[\frac{\frac{1}{p} \int_{\Omega} u^{p} \ln |u| \mathrm{d} x}{\beta} \frac{\beta}{2(\gamma+1)}\|u\|^{2(\gamma+1)}\right] .
$$

We have

$$
\frac{\mathrm{d}^{2}}{\mathrm{~d} \lambda^{2}} \mathcal{J}(\lambda u)=\frac{\beta}{2(\gamma+1)}\|u\|^{2(\gamma+1)}>0
$$

and then $\lambda_{*}$ is a global minimum.

Since $\lambda_{*}<1$, by straight calculation we get $\mathcal{J}\left(\lambda_{*} u\right)<0$, so we introduce the sets

$$
\mathcal{H}_{1}=\left\{u \in H_{0}^{1}(\Omega) ; \mathcal{J}\left(\lambda_{*} u\right) \leq \mathcal{J}(\lambda u) \leq 0\right\}
$$

and

$$
\mathcal{H}_{2}=\left\{u \in H_{0}^{1}(\Omega) ; 0<\mathcal{J}(\lambda u)\right\} .
$$

The potential well is defined by $\mathcal{H}=\left\{u \in H_{0}^{1}(\Omega): \mathcal{J}(u)<d\right\} \cup\{0\}$ and partition it into two sets

$$
\begin{aligned}
& V=\left\{u \in \mathcal{H}: \frac{\lambda \beta}{2(\gamma+1)}\|u\|^{2(\gamma+1)}>\frac{1}{p} \int_{\Omega} u^{p} \ln |u| \mathrm{d} x\right\} \cup\{0\}, \\
& W=\left\{u \in \mathcal{H}: \frac{\lambda \beta}{2(\gamma+1)}\|u\|^{2(\gamma+1)}<\frac{1}{p} \int_{\Omega} u^{p} \ln |u| \mathrm{d} x\right\} .
\end{aligned}
$$

We will refer to $V$ as the "good" part of the well and $W$ as the "bad" part of the well. Then we define by $V$ the set of stability for the problem (1)-(3).

\section{TeChnical LEMmas}

In this section, we present hypotheses and lemmas which will need throughout this paper.

Lemma 1. [1,20] Let $c_{q}$ be the smallest positive constant satisfying

$$
\|u\|_{q} \leq c_{q}\|\nabla u\|, \quad \forall u \in H_{0}^{1}(\Omega)
$$

where $2 \leq q<\infty$, if $n=1,2$; and $2 \leq q \leq \frac{2 n}{n-2}$, if $n \geq 3$.

We state general assumptions on $g$ : 
(A1) $g \in C^{1}([0, \infty))$ is a nonincreasing and nonnegative function satisfying

$$
g(0) \geq 0, \alpha-\int_{0}^{\infty} g(s) d s=l>0,
$$

(A2) There exist positive constant $\vartheta$ such that

$$
g^{\prime}(t) \leq \vartheta g(t), t \geq 0 .
$$

The total energy functional $E(t)$ of the solution $u(t)$ of equation (1) is defined as follows:

$$
\begin{aligned}
E(t) & =\frac{1}{2}\left\|u_{t}\right\|^{2}+\frac{1}{2}\left(\alpha-\int_{0}^{t} g(s) \mathrm{d} s\right)\|\nabla u\|^{2}+\frac{1}{p^{2}}\|u\|_{p}^{p} \\
& +\frac{\beta}{2(\gamma+1)}\|\nabla u\|^{2(\gamma+1)}+\frac{1}{2}(g \circ \nabla u)(t)-\frac{1}{p} \int_{\Omega} u^{p} \ln |u| \mathrm{d} x,
\end{aligned}
$$

and we have that $E(t)>0$ in the good part of the well.

Now, we introduce the potential energy functional

$$
\begin{aligned}
J(u) & =\frac{1}{2}\left(\alpha-\int_{0}^{t} g(s) \mathrm{d} s\right)\|\nabla u\|^{2}+\frac{1}{p^{2}}\|u\|_{p}^{p} \\
& +\frac{\beta}{2(\gamma+1)}\|\nabla u\|^{2(\gamma+1)}+\frac{1}{2}(g \circ \nabla u)(t)-\frac{1}{p} \int_{\Omega} u^{p} \ln |u| \mathrm{d} x,
\end{aligned}
$$

and the functional

$$
\begin{aligned}
I(u) & =\left(\alpha-\int_{0}^{t} g(s) \mathrm{d} s\right)\|\nabla u\|^{2}+\beta\|\nabla u\|^{2(\gamma+1)} \\
& +(g \circ \nabla u)(t)-\int_{\Omega} u^{p} \ln |u| \mathrm{d} x,
\end{aligned}
$$

for $u \in H_{0}^{1}(\Omega)$, where

$$
(g \circ \nabla u)(t)=\int_{0}^{t} g(t-s)\|\nabla u(s)-\nabla u(t)\|^{2} \mathrm{~d} s .
$$

Then, it is easy to show that for $u \in H_{0}^{1}(\Omega)$,

$$
\begin{aligned}
J(u) & =\frac{1}{p} I(u)+\frac{(p-2) l}{2 p}\|\nabla u\|^{2}+\frac{p-2 \gamma-2}{2 p(\gamma+1)} \beta\|\nabla u\|^{2(\gamma+1)} \\
& +\frac{(p-2)}{2 p}(g \circ \nabla u)(t)+\frac{1}{p^{2}}\|u\|_{p}^{p},
\end{aligned}
$$




$$
E(t)=\frac{1}{2}\left\|u_{t}\right\|^{2}+J(u) .
$$

Remark 1. From the definition of $E(t)$ with $(20),(A 1)-(A 2)$ and Lemma 1 , we get

$$
\begin{aligned}
E(t) & \geq \frac{1}{2}\left(\alpha-\int_{0}^{t} g(s) \mathrm{d} s\right)\|\nabla u\|^{2}+\frac{1}{2}(g \circ \nabla u)(t)-\frac{1}{p} \int_{\Omega} u^{p} \ln |u| \mathrm{d} x \\
& \geq \frac{1}{2}\left(l\|\nabla u\|^{2}+(g \circ \nabla u)(t)\right)-\frac{1}{p}\|u\|_{p+1}^{p+1} \\
& \geq \frac{1}{p}\left[\frac{p}{2}\left(l\|\nabla u\|^{2}+(g \circ \nabla u)(t)\right)-\|u\|_{p+1}^{p+1}\right] \\
& \geq \frac{1}{p}\left[\frac{p}{2}\left(l\|\nabla u\|^{2}+(g \circ \nabla u)(t)\right)-C_{*}^{(p+1)} l^{\frac{p+1}{2}}\|\nabla u\|_{2}^{p+1}\right] \\
& \geq \frac{1}{p} K\left[\left(l\|\nabla u\|^{2}+(g \circ \nabla u)(t)\right)^{\frac{1}{2}}\right], t \geq 0, \text { where }
\end{aligned}
$$

$$
K(\sigma)=\frac{p}{2} \sigma^{2}-C_{*}^{(p+1)} \sigma^{p+1} \text { and } C_{*}=\frac{c}{\sqrt{l}} .
$$

It is easy to verify that there is a maximum value of $K(\sigma)$ at

$$
\sigma_{1}=\left(\frac{p}{p+1} C_{*}^{-(p+1)}\right)^{\frac{1}{p-1}}
$$

and the maximum value is

$$
E_{1}=\frac{p-1}{2}\left(\frac{p}{p+1}\right)^{\frac{p+1}{p-1}} C_{*}^{-\frac{2(p+1)}{p-1}} .
$$

Now, the following assumptions and lemmas have an important role in the proving of our main results.

Lemma 2. Suppose that $(A 1)$ and $(A 2)$ hold. Then the energy functional $E(t)$ is decresing with respect to $t$ and

$$
E^{\prime}(t)=-\frac{1}{2}\left\|\nabla u_{t}\right\|^{2}+\frac{1}{2}\left[\left(g^{\prime} \circ \nabla u\right)(t)-g(t)\|\nabla u(t)\|^{2}\right] \leq-\frac{1}{2}\left\|\nabla u_{t}\right\|^{2} \leq 0,
$$

where

$$
\left(g^{\prime} \circ \nabla u\right)(t)=\int_{0}^{t} g^{\prime}(t-s) \int_{\Omega}|\nabla u(s)-\nabla u(t)|^{2} \mathrm{~d} x \mathrm{~d} t .
$$


Kanıt. We multiply both sides of (1) by $u_{t}$ and then integrating from 0 to $t$, we have

$$
\begin{aligned}
E(t)-E(0) & =-\frac{1}{2}\left\|\nabla u_{t}\right\|^{2}+\int_{0}^{t} \frac{1}{2}\left[\left(g^{\prime} \circ \nabla u\right)(t)-g(t)\|\nabla u(t)\|^{2}\right] \\
& \leq-\frac{1}{2}\left\|\nabla u_{t}\right\|^{2}
\end{aligned}
$$

which yields (24) by a simple calculation.

Lemma 3. Let $u$ be the solution of problem (1) with initial data satisfies $E(0)<E_{1}$ and $l^{\frac{1}{2}}\left\|\nabla u_{0}\right\|<\sigma_{1}$, then

$$
\left(l\|\nabla u\|^{2}+(g \circ \nabla u)(t)\right)^{\frac{1}{2}}<\sigma_{1}
$$

for $t \in[0, T)$.

Kantt. By (22), we see that

$$
K(\sigma)=\left\{\begin{array}{cr}
>0, & 0<\sigma<\sigma_{1} \\
=0, & \sigma<\sigma_{1} \\
<0, & \sigma>\sigma_{1},
\end{array}\right.
$$

and $\lim _{\sigma \rightarrow \infty} K(\sigma)=-\infty$. We prove Lemma 3 by contradiction. Assume (26) does not satisfy. Then, by the continuity of $u(t)$ about time, there is a $t^{*} \in(0, T)$ to make

$$
\left(l\|\nabla u\|^{2}+(g \circ \nabla u)(t)\right)^{\frac{1}{2}}=\sigma_{1} .
$$

By (21), it follows that

$$
\begin{aligned}
E\left(t^{*}\right) & \geq K\left[\left(l\|\nabla u\|^{2}+(g \circ \nabla u)(t)\right)^{\frac{1}{2}}\right] \\
& =K\left(\sigma_{1}\right) \\
& =E_{1} .
\end{aligned}
$$

But this is imposible by $E(t) \leq E(0)<E_{1}$, for $\forall t \geq 0$. Therefore, the proof is completed.

Remark 2. From $(26)$ and $\sigma_{1}=\left(\frac{p}{p+1} C_{*}^{-(p+1)}\right)^{\frac{1}{p-1}}=\left(\frac{p}{p+1}\right)^{\frac{1}{p-1}} c^{-\frac{(p+1)}{p-1}} l^{\frac{2(p+1)}{p-1}}$, we obtain

(28) $l\|\nabla u\|^{2} \leq l\|\nabla u\|^{2}+(g \circ \nabla u)(t)<\sigma_{1}^{2}=\left(\frac{p}{p+1}\right)^{\frac{2}{p-1}} l^{\frac{4(p+1)}{p-1}} c^{-\frac{2(p+1)}{p-1}}$, 
which implies that

(29)

$$
\begin{aligned}
I(u) & =\left(\alpha-\int_{0}^{t} g(s) \mathrm{d} s\right)\|\nabla u\|^{2}+\|\nabla u\|^{2(\gamma+1)}+(g \circ \nabla u)(t)-\int_{\Omega} u^{p} \ln |u| \mathrm{d} x \\
& \geq\left(\alpha-\int_{0}^{\infty} g(s) \mathrm{d} d s\right)\|\nabla u\|^{2}+\|\nabla u\|^{2(\gamma+1)}+(g \circ \nabla u)(t)-\int_{\Omega} u^{p} \ln |u| \mathrm{d} x \\
& \geq l\|\nabla u\|^{2}-\|u\|_{p+1}^{p+1} \\
& \geq l\|\nabla u\|^{2}-c^{p+1}\|\nabla u\|_{2}^{p+1} \geq 0 .
\end{aligned}
$$

Further, by (19), we get

$$
\begin{aligned}
J(u) & =\frac{1}{p} I(u)+\frac{(p-2) l}{2 p}\|\nabla u\|^{2}+\frac{p-2 \gamma-2}{2 p(\gamma+1)} \beta\|\nabla u\|^{2(\gamma+1)} \\
& +\frac{p-2}{2 p}(g \circ \nabla u)(t)+\frac{1}{p^{2}}\|u\|_{p}^{p} \\
& \geq \frac{p-2}{2 p}\left[l\|\nabla u\|^{2}+(g \circ \nabla u)(t)\right]+\frac{1}{p} I(u) .
\end{aligned}
$$

By (29), (30), (20) and Lemma 2 we conclude that

$$
l\|\nabla u\|^{2} \leq \frac{2 p}{p-2} J(u) \leq \frac{2 p}{p-2} E(t) \leq \frac{2 p}{p-2} E(0) \leq \frac{2 p}{p-2} E_{1} .
$$

Since $\log x<x$ for any $x>0$, using Lemma 1 , we get that

$$
\begin{aligned}
\int_{\Omega} u^{p} \ln |u| \mathrm{d} x & \leq\|u\|_{p+1}^{p+1} \\
& \leq c^{p+1}\|\nabla u\|^{p+1} \\
& \leq \frac{c^{p+1}}{l}\left(\frac{2 p}{l(p-2)} E(0)\right)^{\frac{p-1}{2}} l\|\nabla u\|^{2} \\
& =\mu l\|\nabla u\|^{2} \\
& \leq \frac{2 p \mu}{p-2} E(t),
\end{aligned}
$$

where $\mu=\frac{c^{p+1}}{l}\left(\frac{2 p}{l(p-2)} E(0)\right)^{\frac{p-1}{2}}$. Notice that $E(0)<E_{1}$ if and only if

$$
\mu=\frac{c^{p+1}}{l}\left(\frac{2 p}{l(p-2)} E(0)\right)^{\frac{p-1}{2}}<1 .
$$




\section{Global existence}

We start this section, present the local existence theorem. The proof of theorem 1 can be found similar to [14,23].

Theorem 1. Let $u_{0} \in W, u_{1} \in H_{0}^{1}(\Omega)$. Assume that $(A 1), E(0)<E_{1}$ and $l\|\nabla u\|^{2} \leq \sigma_{1}^{2}$ hold. Then problem (1) has a unique solution $u(x, t)$ satisfies $u(t) \in L^{\infty}\left(0, T ; H_{0}^{1}(\Omega)\right)$ with $u_{t}(t) \in L^{\infty}\left(0, T ; H_{0}^{1}(\Omega)\right)$.

Moreover, at least one of the following statements holds true, $T=\infty$ or $\left\|u_{t}\right\|^{2}+\|\Delta u\|^{2} \rightarrow \infty$ as $t \rightarrow T^{-}$.

Now we are in position to state and prove the global existence result.

Theorem 2. Let $u_{0} \in W, u_{1} \in H_{0}^{1}(\Omega)$. Suppose that $(A 1), 0<E(0)<E_{1}$ and $l\|\nabla u\|^{2} \leq \sigma_{1}^{2}$ hold. Then problem (1)-(3) admits a global weak solution $u(t) \in L^{\infty}\left(0, \infty ; H_{0}^{1}(\Omega)\right)$ with $u_{t}(t) \in L^{\infty}\left(0, \infty ; H_{0}^{1}(\Omega)\right)$.

Kanıt. Our aim is to show that the solutions exist when $T=\infty$. First, multiplying (1) by $-2 \Delta u$, and integrating it over $\Omega$, we obtain

$$
\begin{aligned}
& \frac{d}{d t}\left\{\|\Delta u\|^{2}-2 \int_{\Omega} u_{t} \Delta u \mathrm{~d} x\right\}+2 M\left(\|\nabla u\|^{2}\right)\|\Delta u\|^{2} \\
& \leq 2\left\|\nabla u_{t}\right\|^{2}-2 \int_{\Omega}|u|^{p-2} u \ln |u| \Delta u \mathrm{~d} x+2 \int_{0}^{t} g(t-s) \int_{\Omega} \Delta u(s) \Delta u(t) \mathrm{d} x \mathrm{~d} s .
\end{aligned}
$$

We now estimate the last term in the right side of (35) as follows

$2 \int_{0}^{t} g(t-s) \int_{\Omega} \triangle u(s) \Delta u(t) \mathrm{d} x \mathrm{~d} s \leq 2 \varepsilon\|\Delta u\|^{2}+\frac{\|g\|_{L^{1}}}{2 \varepsilon} \int_{0}^{t} g(t-s)\|\Delta u(s)\|^{2} \mathrm{~d} s$.

where $0<\varepsilon<\frac{\|g\|_{L^{1}}}{2}$.

Thus, by inserting (36) in (35) and then multiplying it by $\eta, 0<\eta \leq 1$, we have

$$
\begin{aligned}
& \frac{\mathrm{d}}{\mathrm{d} t}\left\{\eta\|\Delta u\|^{2}-2 \eta \int_{\Omega} u_{t} \Delta u \mathrm{~d} x\right\}+2 \eta\left(M\left(\|\nabla u\|^{2}\right)-\varepsilon\right)\|\Delta u\|^{2} \\
& \leq 2 \eta\left\|\nabla u_{t}\right\|^{2}-2 \eta \int_{\Omega}|u|^{p-2} u \ln |u| \Delta u \mathrm{~d} x+\eta \frac{\|g\|_{L^{1}}}{2 \varepsilon} \int_{0}^{t} g(t-s)\|\Delta u(s)\|^{2} \mathrm{~d} s .
\end{aligned}
$$


Then adding $2 E^{\prime}(t)$ to $(37)$, we have

$$
\begin{aligned}
& \frac{\mathrm{d}}{\mathrm{d} t} E^{*}(t)+2 \eta\left(M\left(\|\nabla u\|^{2}\right)-\varepsilon\right)\|\Delta u\|^{2}+2(1-\eta)\left\|\nabla u_{t}\right\|^{2} \\
& \leq-2 \eta \int_{\Omega}|u|^{p-2} u \ln |u| \Delta u \mathrm{~d} x+\eta \frac{\|g\|_{L^{1}}}{2 \varepsilon} \int_{0}^{t} g(t-s)\|\Delta u(s)\|^{2} \mathrm{~d} s
\end{aligned}
$$

where

$$
E^{*}(t)=2 E(t)+\eta\|\Delta u\|^{2}-2 \eta \int_{\Omega} u_{t} \Delta u \mathrm{~d} x .
$$

Applying Young's inequality, we obtain

$$
\left|2 \eta \int_{\Omega} u_{t} \Delta u \mathrm{~d} x\right| \leq 2 \eta\left\|u_{t}\right\|^{2}+\frac{\eta}{2}\|\Delta u\|^{2} .
$$

By Remark 2 and (30) we see clearly that $J(t) \geq 0$, then by (20) we get

$$
\left\|u_{t}\right\|^{2} \leq 2 E(t) \text {. }
$$

By using of (40) and (41), (39) becomes

$$
\begin{aligned}
E^{*}(t) & \geq(1-2 \eta)\left\|u_{t}\right\|^{2}+\frac{\eta}{2}\|\Delta u\|^{2} \\
& =\frac{1}{5}\left(\left\|u_{t}\right\|^{2}+\|\Delta u\|^{2}\right), \text { where } \eta=\frac{2}{5} .
\end{aligned}
$$

Moreover, we notice that

$$
\begin{aligned}
\left.2\left|\int_{\Omega}\right| u\right|^{p-2} \ln |u| u \Delta u \mathrm{~d} x \mid & \leq \int_{\Omega}|u|^{p-2} \ln |u||\nabla u|^{2} \mathrm{~d} x \\
& \leq \int_{\Omega}|u|^{p-1}|\nabla u|^{2} \mathrm{~d} x \\
& \leq\|u\|_{(p-1) \theta_{1}}^{p-1}\|\nabla u\|_{2 \theta_{2}}^{2},
\end{aligned}
$$

where $\frac{1}{\theta_{1}}+\frac{1}{\theta_{2}}=1$, so that, we put $\theta_{1}=1$ and $\theta_{2}=\infty$, if $n=1 ; \theta_{1}=1+\varepsilon_{1}$, if $n=2$; and $\theta_{1}=\frac{n}{2}, \theta_{2}=\frac{n}{n-2}$, if $n \geq 3$. Then, by using Lemma 1, we have

$$
\left.2\left|\int_{\Omega}\right| u\right|^{p-2} \ln |u| u \Delta u \mathrm{~d} x \mid \leq c_{s}^{p}\|\nabla u\|_{2}^{p-1}\|\Delta u\|^{2} .
$$

By (31) and (42), we obtain

$$
\left.2\left|\int_{\Omega}\right| u\right|^{p-2} \ln |u| u \Delta u \mathrm{~d} x \mid \leq c_{1} E^{*}(t),
$$


where $c_{1}=5 c_{s}^{p}\left(\frac{2 p}{l(p-2)} E(0)\right)^{\frac{p-1}{2}}$.

Substituting (43) into (38), and then integrating it over $(0, t)$, we get

$$
\begin{aligned}
& E^{*}(t)+\frac{4}{5}\left(\alpha-\varepsilon-\frac{\|g\|_{L^{1}}^{2}}{4 \varepsilon}\right) \int_{0}^{t}\|\Delta u(s)\|^{2} \mathrm{~d} s \\
& \leq E^{*}(0)+\int_{0}^{t} c_{1} E^{*}(s) d s,
\end{aligned}
$$

hence by choosing $\varepsilon=\frac{\|g\|_{L^{1}}}{2}$ we see that

$$
E^{*}(t) \leq E^{*}(0)+\int_{0}^{t} c_{1} E^{*}(s) \mathrm{d} s .
$$

Then, by using of Gronwall's inequality, we arrive at

$$
E^{*}(t) \leq E^{*}(0) e^{c_{1} t},
$$

for any $t \geq 0$. Therefore by (42) and Theorem 1 , we obtain $T=\infty$.

\section{BLOW UP}

In this section, we prove the blow up result of solution for the problem (1)-(3), taking into account that the initial data is localized in instability set.

Theorem 3. Suppose that $u_{0} \in W$ and $u_{1} \in H_{0}^{1}(\Omega)$ and Lemma 2 holds. Then the solution of the equation (1) blows up as time $t$ goes to infinity as long as $E(0)<0$ and $I\left(u_{0}\right)>0$.

Kantt. Our aim is to show that the finite time blow up of solution to equation (1). If it is not case, we suppose that the solution $u(x, t)$ is global. For any $T_{0}>0$, defining the following auxiliary function

$$
G(t)=\|u\|^{2}+\int_{0}^{t}\|\nabla u(\tau)\|^{2} \mathrm{~d} \tau+\left(T_{0}-t\right)\left\|\nabla u_{0}\right\|^{2},
$$

then we see clear that for $G(t)>0$ for all $t \in\left[0, T_{0}\right]$. It is obvious that $G(t)>0$. In view of the continuity of $G(t)$ in $t$, we obtain that there is a $\rho>0$ such that

$$
G(t) \geq \rho,
$$

where $\rho$ is independent of $T_{0}$.

Then by $t \in\left[0, T_{0}\right]$, we have

$$
G^{\prime}(t)=2\left(u, u_{t}\right)+\|\nabla u\|^{2}-\left\|\nabla u_{0}\right\|^{2}
$$




$$
=2\left(u, u_{t}\right)+\int_{0}^{t}\left(\nabla u(\tau), \nabla u_{\tau}(\tau)\right) \mathrm{d} \tau
$$

and

$$
\begin{aligned}
G^{\prime \prime}(t) & =2\left\|u_{t}\right\|^{2}+2 \int_{\Omega} u_{t t} u \mathrm{~d} x+2\left(\nabla u, \nabla u_{t}\right) \\
& =2\left\|u_{t}\right\|^{2}-2 \int_{\Omega} M\left(\|\nabla u\|^{2}\right)\|\nabla u\|^{2} \mathrm{~d} x \\
& +2 \int_{0}^{t} g(t-s) \int_{\Omega} \nabla u(s) \nabla u(t) \mathrm{d} s \mathrm{~d} x+2 \int_{\Omega} u^{p} \ln |u| \\
& =2\left\|u_{t}\right\|^{2}-\left(\alpha-\int_{0}^{t} g(s) \mathrm{d} s\right)\|\nabla u\|^{2}-2\|\nabla u\|^{2(\gamma+1)} \\
& +\int_{0}^{t} g(t-s)\|\nabla u(s)\|^{2} \mathrm{~d} s-(g \circ \nabla u)(t)+2 \int_{\Omega} u^{p} \ln |u| \\
\geq & 2\left\|u_{t}\right\|^{2}-2 I(u) .
\end{aligned}
$$

By (44), we see that

$$
\begin{aligned}
\left(G^{\prime}(t)\right)^{2} & =4\left[\left(u, u_{t}\right)^{2}+\left(\int_{0}^{t}\left(\nabla u(\tau), \nabla u_{\tau}(\tau)\right) \mathrm{d} \tau\right)^{2}\right] \\
& +4\left(u, u_{t}\right) \int_{0}^{t}\left(\nabla u(\tau), \nabla u_{\tau}(\tau)\right) \mathrm{d} \tau .
\end{aligned}
$$

Now we shall estimate the each terms in (46) by Cauchy-Schwarz, Hölder and Young's inequalities as follows

$$
\left(\int_{0}^{t}\left(\nabla u(\tau), \nabla u_{\tau}(\tau)\right) \mathrm{d} \tau\right)^{2} \leq \int_{0}^{t}\|\nabla u\|^{2} \mathrm{~d} \tau \int_{0}^{t}\left\|\nabla u_{\tau}\right\|^{2} \mathrm{~d} \tau .
$$

By combining (47) and (48) and using Young's inequality, we have

$$
2\left(u, u_{t}\right) \int_{0}^{t}\left(\nabla u(\tau), \nabla u_{\tau}(\tau)\right) \mathrm{d} \tau
$$




$$
\begin{aligned}
& \leq 2\|u\|\left\|u_{t}\right\|\left(\int_{0}^{t}\|\nabla u\|^{2} d \tau\right)^{\frac{1}{2}}\left(\int_{0}^{t}\left\|\nabla u_{\tau}\right\|^{2} \mathrm{~d} \tau\right)^{\frac{1}{2}} \\
& \leq\|u\|^{2} \int_{0}^{t}\left\|\nabla u_{\tau}\right\|^{2} \mathrm{~d} \tau+\left\|u_{t}\right\|^{2} \int_{0}^{t}\|\nabla u\|^{2} \mathrm{~d} d \tau .
\end{aligned}
$$

Inserting (47)-(49) into (46) becomes

$$
\begin{aligned}
\left(G^{\prime}(t)\right)^{2} \leq & 4\|u\|^{2}\left\|u_{t}\right\|^{2}+4 \int_{0}^{t}\|\nabla u\|^{2} \mathrm{~d} \tau \int_{0}^{t}\left\|\nabla u_{\tau}\right\|^{2} \mathrm{~d} d \tau \\
& +4\left(\|u\|^{2} \int_{0}^{t}\left\|\nabla u_{\tau}\right\|^{2} \mathrm{~d} \tau+\left\|u_{t}\right\|^{2} \int_{0}^{t}\|\nabla u\|^{2} \mathrm{~d} \tau\right) \\
& =4\|u\|^{2}\left(\left\|u_{t}\right\|^{2}+\int_{0}^{t}\left\|\nabla u_{\tau}\right\|^{2} \mathrm{~d} \tau\right) \\
& +4 \int_{0}^{t}\|\nabla u\|^{2} \mathrm{~d} d \tau\left(\left\|u_{t}\right\|^{2}+\int_{0}^{t}\left\|\nabla u_{\tau}\right\|^{2} \mathrm{~d} \tau\right) \\
& =4\left(\|u\|^{2}+\int_{0}^{t}\|\nabla u\|^{2} \mathrm{~d} \tau\right)\left(\left\|u_{t}\right\|^{2}+\int_{0}^{t}\left\|\nabla u_{\tau}\right\|^{2} \mathrm{~d} \tau\right) \\
& \leq 4 G(t)\left(\left\|u_{t}\right\|^{2}+\int_{0}^{t}\left\|\nabla u_{\tau}\right\|^{2} \mathrm{~d} \tau\right) .
\end{aligned}
$$

Then by (45) and (50) we deduce

$$
\begin{aligned}
& G^{\prime \prime}(t) G(t)-\frac{p+2}{4}\left(G^{\prime}(t)\right)^{2} \\
& \geq G(t)\left[G^{\prime \prime}(t)-(p+2)\left(\left\|u_{t}\right\|^{2}+\int_{0}^{t}\left\|\nabla u_{\tau}\right\|^{2} \mathrm{~d} \tau\right)\right] \\
& \geq G(t)\left[2\left\|u_{t}\right\|^{2}-2 I(u)-(p+2)\left(\left\|u_{t}\right\|^{2}+\int_{0}^{t}\left\|\nabla u_{\tau}\right\|^{2} \mathrm{~d} \tau\right)\right] \\
& =G(t) \xi(t),
\end{aligned}
$$


where

$$
\xi(t)=-p\left\|u_{t}\right\|^{2}-2 I(u)-(p+2) \int_{0}^{t}\left\|\nabla u_{\tau}\right\|^{2} \mathrm{~d} \tau .
$$

By Lemma 2 and (19) we obtain

$$
\begin{aligned}
E(0) \geq & \int_{0}^{t}\left\|\nabla u_{\tau}\right\|^{2} \mathrm{~d} \tau+E(t) \\
= & \int_{0}^{t}\left\|\nabla u_{\tau}\right\|^{2} \mathrm{~d} \tau+\frac{1}{2}\left\|u_{t}\right\|^{2}+J(u) \\
= & \int_{0}^{t}\left\|\nabla u_{\tau}\right\|^{2} \mathrm{~d} \tau+\frac{1}{2}\left\|u_{t}\right\|^{2}+\frac{1}{p} I(u)+\frac{(p-2) l}{2 p}\|\nabla u\|^{2} \\
& +\frac{p-2 \gamma-2}{2 p(\gamma+1)} \beta\|\nabla u\|^{2(\gamma+1)}+\frac{(p-2)}{2 p}(g \circ \nabla u)(t)+\frac{1}{p^{2}}\|u\|_{p}^{p} .
\end{aligned}
$$

Inserting (53) into (52) we obtain

$$
\begin{aligned}
\xi(t) & >(p-2) \int_{0}^{t}\left\|\nabla u_{\tau}\right\|^{2} \mathrm{~d} \tau-2 p E(0)+(p-2) l\|\nabla u\|^{2} \\
& +\frac{p-2 \gamma-2}{(\gamma+1)} \beta\|\nabla u\|^{2(\gamma+1)}+(p-2)(g \circ \nabla u)(t)+\frac{1}{p^{2}}\|u\|_{p}^{p} .
\end{aligned}
$$

Under the condition $E(0)<0$, we obtain

$$
(p-2) l\|\nabla u\|^{2}-2 p E(0)>0 .
$$

Hence by (55) we arrive at that

$$
\begin{aligned}
\xi(t) & >(p-2)\left(\int_{0}^{t}\left\|\nabla u_{\tau}\right\|^{2} \mathrm{~d} \tau+(g \circ \nabla u)(t)\right) \\
& +\frac{p-2 \gamma-2}{(\gamma+1)} \beta\|\nabla u\|^{2(\gamma+1)}+\frac{1}{p^{2}}\|u\|_{p}^{p} \\
& >\vartheta>0 .
\end{aligned}
$$

Then, by using (56), we can see clearly that

$$
G^{\prime \prime}(t) G(t)-\frac{p+2}{4}\left(G^{\prime}(t)\right)^{2} \geq G(t) \vartheta .
$$

Let $y(t)=G(t)^{\frac{-p+2}{4}}$, then we obtain

$$
y^{\prime \prime}(t) \leq-\frac{p-2}{4} \vartheta y(t)^{\frac{p+2}{p-2}}, t \in\left[0, T_{0}\right]
$$


That is

$$
\lim _{t \rightarrow T^{*}} y(t)=0,
$$

where $T^{*}$ is independent of initial choice of $T_{0}$ and $T^{*}<T_{0}$. Therefore we can conclude that

$$
\lim _{t \rightarrow T^{*}} G(t)=0 .
$$

Acknowledgment. The authors thank the referees for their valuable considerations, which is improved this manuscript.

\section{REFERENCES}

[1] R.A. Adams, J.J.F. Fournier, Sobolev Spaces, Academic Press, 2003.

[2] M.M. Al-Gharabli, A. Guesmia, S.A. Messaoudi, Well posedness and asymptotic stability results for a viscoelastic plate equation with a logarithmic nonlinearity, Applicable Analysis, 99 (1) (2020), 50-74.

[3] A. Ambrosetti, P.H. Rabinowitz, Dual variational methods in critical point theory and applications, Journal of Functional Analysis, 14 (1973), 349-381.

[4] S. Boulaaras, A. Draifia, M. Alnegga, Polynomial decay rate for Kirchhoff type in viscoelasticity with logarithmic nonlinearity and not necassarily decreasing kernel, Symmetry, 11 (2) (2019), 1-24.

[5] I. Bialynicki-Birula, J. Mycielski, Wave equations with logarithmic nonlinearities, Bulletin L'Académie Polonaise des Science, Série des Sciences Mathématiques, Astronomiques et Physiques, 23 (4) (1975), 461-466.

[6] H. Buljan, A. Siber, M. Soljacic, T. Schwartz, M. Segev, D.N. Christodoulides, Incoherent white light solitons in logarithmically saturable noninstantaneous nonlinear media, Physical Review E, 68 (3) (2003), 1-6.

[7] X. Han, Global existence of weak solutions for a logarithmic wave equation arising from Q-ball dynamics, Bulletin of the Korean Mathematical Society, 50 (1) (2013), $275-283$.

[8] Z. Hongwei, L. Gongwei, H. Qingying, Exponential decay of energy for a logarithmic wave equation, Journal of Partial Differential Equations, 28 (3) (2015), 269-277.

[9] Q. Hu, H. Zhang, G. Liu, Asymptotic behavior for a class of logarithmic wave equations with linear damping, Applied Mathematics \& Optimization, 79 (2019), 131-144.

[10] G. Kirchhoff, Vorlesungen über Mechanik, Leipzig, Tauber, 1883.

[11] W. Krolikowski, D. Edmundson, O. Bang, Unified model for partially coherent solitons in logarithmically nonlinear media, Physical Review E, 61 (2000), 3122-3126.

[12] W. Lian, M.S. Ahmed, R. Xu, Global existence and blow up of solution for semilinear hyperbolic equation with logarithmic nonlinearity, Nonlinear Analysis, 184 (2019), 239-257. 
[13] A. Linde, Strings, Textures, inflation and spectrum bending, Physics Letters B, 284 (1992), 215-222.

[14] G. Liu, The existence, general decay and blow up for a plate equation with nonlinear damping and a logarithmic source term, Electronic Research Archive, 28 (1) (2020), 263-289.

[15] L. Ma, Z.B. Fang, Energy decay estimates and infinite blow-up phenomena for a strongly damped semilinear wave equation with logarithmic nonlinear source, Mathematical Methods in the Applied Sciences, 41 (2018), 2639-2653.

[16] S.A. Messaoudi, M.M. Al-Gharabli, A. Guesmia, Existence and a general decay results for a viscoelastic plate equation with a logarithmic nonlinearity, Communications on Pure \& Applied Analysis, 18 (1) (2019), 159-180.

[17] N. Mezouar, S.M. Boulaaras, A. Allahem, Global existence of solutions for the viscoelastic Kirchhoff equation with logarithmic source terms, Complexity, (2020), Article ID: $7105387,1-25$.

[18] D.C. Pereira, C.A. Raposo, C.H.M. Maranhão, A.P Cattai, Global existence and uniform decay of solutions for a Kirchhoff beam equation with nonlinear damping and source term, Differential Equations and Dynamical Systems, (2021).

[19] A. Peyravi, General stability and exponential growth for a class of semi-linear wave equations with logarithmic source and memory terms, Applied Mathematics \& Optimization, 81 (2020), 545-561.

[20] E. Pişkin, Sobolev Spaces, Seçkin Publishing, Turkish, 2017.

[21] E. Pişkin, N. Irkıl, Exponential growth of solutions of higher-order viscoelastic wave equation with logarithmic term, Erzincan Binali Yıldırım University Journal of Science and Technology, 13 (1) (2020), 106-111.

[22] M. Willem, "Minimax Theorems", Progress in Nonlinear Differential Equations and their Applications, 24, Birkhöuser Boston Inc., Boston, 1996.

[23] S.T. Wu, L.Y. Tsai, On global existence and blow-up of solutions for an integrodifferential equation with strong damping, Taiwanese Journal of Mathematics, 10 (4) (2006), 979-1014.

[24] Y. Ye, Global existence and asymptotic behavior of solutions for a class of nonlinear degenerate wave equations, Differential Equations and Nonlinear Mechanics, (2007), Article ID: 019685, 10 pages.

[25] Y. Ye, Logarithmic viscoelastic wave equation in threedimensional space, Applicable Analysis, (2019), 1-18. 


\section{JoRge FERREIRA}

Department of Exact Sciences

Federal Fluminense University

27255-435 Volta Redonda, RJ

BRASIL

E-mail address: ferreirajorge2012@gmail.com

\section{ERHAN PişKin}

Department of Mathematics

Dicle University

TR-21280 DiYARBAKIR

TURKEY

E-mail address: episkin@dicle.edu.tr

Nazli Irkil

Department of Mathematics

Dicle University

TR-21280 DIYARBAKIR

TURKEY

E-mail address: nazliirkil@gmail.com

\section{Carlos Raposo}

Department of Mathematics

Federal University of SÃo João del-Rei 36307-352 SÃO JoÃo DEL-REI, MG

BRAZIL

E-mail address: raposo@ufsj.edu.br 